\title{
SINOPSE DOS CRUSTÁCEOS DECÁPODOS BRASILEIROS (PORTUNIDAE)
}

\author{
Petrônio Alves Coelho ${ }^{1}$ \\ Marilena Ramos-Porto ${ }^{2}$
}

\begin{abstract}
SYNOPSIS OF THE BRAZILIAN DECAPOD CRUSTACEANS (PORTUNIDAE). This paper presents a contribution to the study of the Crustacea, Decapoda of Brazil, including identification keys and informations on species range. Data related to the species of family Portunidae are published.
\end{abstract}

KEY WORDS. Crustacea, Decapoda, Portunidae, sinopsis, Brazil

A "Sinopse dos crustáceos decápodos brasileiros" divulga os dados existentes, principalmente nos Departamentos de Oceanografia da Universidade Federal do Pernambuco e de Pesca da Universidade Federal Rural de Pernambuco, relativamente à fauna de crustáceos decápodos do Brasil. Desta forma, são estudadas famílias não só marinhas, como também de água doce ou até terrestres. Além das espécies de ocorrência confirmada no Brasil, eventualmente são incluídas outras, cuja presença seja suspeitada em função de indícios de natureza oceanográfica ou potamográfica. Em vários casos, estão incluídos dados obtidos pelos autores estudando coleções pertencentes a outras instituições, notadamente ao Museu de Zoologia da Universidade de São Paulo ou ao Museu Nacional da Universidade Federal do Rio de Janeiro.

Os trabalhos anteriores da série se referem às famílias Penaedidae, Solenoceridae, Sicyoniidae, Palaemonidae, Scyllaridae, Palinuridae, Nephropidae, Parastacidae, Axiidae, Callianideidae, Callianassidae, Upogebiidae, Paguridae, Parapaguridae, Pylochelidae, Diogenidae, Hippidae, Abuneidae, Dromiidae, Homolidae, Leucosiidae e Dorippidae. Em continuação, é apresentada a sinopse dos Portunidae.

\section{MATERIAL E MÉTODOS}

O trabalho está constituído essencialmente por chaves de identificação para subfamílias, gêneros e espécies encontradas no Brasil. Para cada espécie, estão mencionados o nome científico válido, algumas referências básicas do

1) Departamento de Oceanografia, Universidade Federal de Pernambuco, Av. Prof. Moraes Rêgo, Cidade Universitária, 50670-420 Recife, Pernambuco, Brasil. Bolsista do CNPq.

2) Departamento de Pesca, Universidade Federal Rural de Pernambuco, Rua Dom Manoel de Medeiros, 52097-420 Recife, Pernambuco, Brasil. Bolsista do CNPq. 
ponto de vista taxonômico ou biogeográfico, dados de coleta do material examinado e distribuição geográfica. Estão assinaladas com um asterisco as espécies não referidas por COELHO \& RAMOS (1972); para as demais, foi considerado desnecessário mencionar o referido trabalho.

\section{Portunidae Rafinesque, 1815}

Chave para identificação das subfamílias

1. Carapaça estreita; três ou cinco dentes ântero-laterais .......... Polybiinae - Carapaça larga; nove dentes ântero-laterais ................ Portuninae

\section{Polybiinae Ortmann, 1893}

Chave para identificação dos gêneros

1. Cinco dentes ântero-laterais; antenas não excluídas das órbitas .... Ovalipes -Três dentes ântero-laterais; antenas excluídas das órbitas.... Coenophthalmus

\section{Ovalipes Rathbun, 1898}

\section{Ovalipes trimaculatus (De Haan, 1833)*}

STEPHENSON \& REIS, 1968:20.

Material. Rio Grande do Sul; 25m, infralitoral.

Distribuição geográfica. Atlântico Ocidental: desde o Rio Grande do Sul até Chubut; Pacífico Oriental: Peru, Chile; Atlântico Central: Tristão da Cunha; Índico Ocidental: África do Sul.

Observação. SEBA (1759) publicou a ilustração de um Portunidae identificada como "Ciri poa, seu Aratu Pinima, Brasiliensis", o qual foi colocado por RATHBUN (1930), na sinonímia de Ovalipes ocellatus guadulpensis (Saussure, 1858), isto é, O. floridensis Hay \& Shore, 1918, que ainda não foi encontrada no Brasil. Segundo HOLTHUIS (1991) a identificação da figura de SEBA (1759) deve ser corrigida para Liocarcinus holsatus (Fabricius, 1798), que ocorre apenas no Atlântico Norte-Oriental.

Coenophthalmus A. Milne Edwards, 1879

\section{Coenophthalmus tridentatus A. Milne Edwards, 1879}

BOSCHI, 1964: 47.

Material. Rio de Janeiro; Argentina, Buenos Aires, 48m infralitoral.

Distribuição geográfica. Atlântico Ocidental: desde o Rio de Janeiro até Chubut. 


\section{Portuninae Rafinesque, 1815}

Chave para identificação dos gêneros

1. Flagelo das antenas excluído das órbitas por um prolongamento do artículo basal; dentes ântero-laterais alternadamente grandes e pequenos ...... Cronius

- Flagelo das antenas não excluído das órbitas; dentes ântero-laterais de tamanho semelhante, exceto o posterior, ou dente lateral ............2

2. Fissuras supra-orbitais em forma de "U" aberto; palato desprovido de carenas longitudinais .....................................

- Fissuras supra-orbitais fechadas; palato com carenas longitudinais .3

3. Carpo das quelas com dois espinhos (um interno e outro externo)... Portunus Aranaeus Dana, 1851

Aranaeus cribrarius (Lamarck, 1818)

WILLIAMS, 1984:302.

Material. Rio Grande do Norte, Paraíba, Pernambuco, Alagoas, Sergipe, São Paulo, Rio Grande do Sul; recifes; fundos de areia; 0-25m, infralitoral.

Distribuição geográfica. Atlântico Ocidental: desde Massachussetts até a Venezuela e desde o Ceará até o Rio Grande do Sul.

Callinectes Stimpson, 1860

Chave para identificação das espécies

1. Dois dentes na fronte, situados entre os dentes do bordo interno das órbitas sapidus

- Quatro dentes na fronte, situados entre os dentes do bordo interno das órbitas

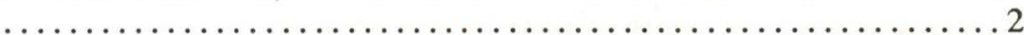

2. Dentes laterais da carapaça pouco desenvolvidos; dentes da fronte pouco diferentes no tamanho ........................... 3

- Dentes laterais da carapaça muito longos; dentes internos da órbita com menos da metade do comprimento dos dentes externos .......... 4

3. Regiões ântero-laterais da carapaça lisas ao tato; pleópodos do macho adulto atingindo a extremidade do abdômen ................. bocourti

- Regiões ântero-laterais da carapaça ásperas ao tato; pleópodos do macho adulto atingindo a metade do penúltimo segmento do abdômen ....... 
4. Todos os dentes ântero-laterais, exceto os dois primeiros, com o ápice curvado

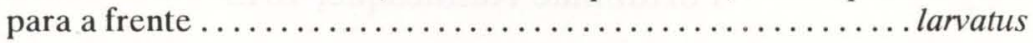

- Apenas o último ou os dois últimos dentes ântero-laterais com o ápice voltado

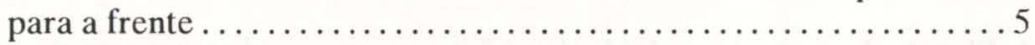

5. Área central trapezoidal da carapaça de largura igual a aproximadamente três vezes o seu comprimento ...................... ornatus

- Área central trapezoidal da carapaça de largura igual a aproximadamente duas vezes e meia o seu comprimento .................. danae

\section{Callinectes bocourti A. Milne Edwards, 1879}

WILLIAMS, 1984: 365.

Material. Amapá, Pernambuco, Alagoas; estuários; prados de Halodule; infralitoral.

Distribuição geográfica. Atlântico Ocidental: desde a Flórida até Santa Catarina.

\section{Callinectes danae Smith, 1869}

WILLIAMS, 1984: 367.

Material. Rio Grande do Norte, Paraíba, Pernambuco, Alagoas; estuários; recifes, fundos de lama, de areia ou de algas calcárias; $0-36 \mathrm{~m}$, infralitoral.

Distribuição geográfica. Atlântico Ocidental: desde a Carolina do Norte até Santa Catarina; Atlântico Central: Bermudas.

Callinectes exasperatus (Gerstaecker, 1856)

WILLIAMS, 1984: 369.

Material. Rio Grande do Norte, Pernambuco; estuários; infralitoral.

Distribuição geográfica. Atlântico Ocidental: desde a Flórida até Santa Catarina.

\section{Callinectes larvatus Ordway, 1863}

Callinectes marginatus; COELHO \& RAMOS, 1972: 187; WILLIAMS, 1984: 371.

Material. Rio Grande do Norte, Paraíba, Pernambuco, Alagoas; estuários; recifes; fundos de areia ou de lama; infralitoral.

Distribuição geográfica. Atlântico Ocidental: desde a Carolina do Norte até São Paulo; Atlântico Central: Bermudas.

\section{Callinectes ornatus Ordway, 1863}

WILLIAMS, 1984: 373.

Material. Amapá, Pará, Maranhão, Ceará, Rio Grande do Norte, Pernambuco, Alagoas, Sergipe, Bahia, Rio Grande do Sul; estuários; recifes; fundos 
de lama, de areia ou de algas calcárias; 0-70m, infralitoral e circalitoral.

Distribuição geográfica. Atlântico Ocidental: desde a Virgínia até o Rio Grande do Sul; Atlântico Central: Bermudas.

\section{Callinectes spidus Rathbun, 1896}

WILLIAMS, 1984: 376.

Material. Rio Grande do Sul.

Distribuição geográfica. Atlântico Ocidental: desde a Nova Escócia até o Uruguai; Atlântico Central: Bermudas; Atlântico Oriental: desde a Dinamarca até o Mediterrâneo; Pacífico Oriental: Japão.

\section{Cronius Stimpson, 1860}

Chave para identificação das espécies

1. Mão das quelas armadas com quatro espinhos $\ldots \ldots \ldots \ldots \ldots \ldots$ ruber

2. Mão das quelas armadas com dois espinhos ...............tumidulus

\section{Cronius ruber (Lamarck, 1818)}

WILLIAMS, 1984: 385.

Material. Amapá, Maranhão, Ceará; fundos de areia, de algas calcárias ou detríticos; 17-105m, infralitoral e circalitoral.

Distribuição geográfica. Atlântico Ocidental: desde Nova Jersey até Santa Catarina; Atlântico Central: Ilhas do Cabo Verde, Príncipe, São Tomé, Ano Bom; Atlântico Oriental: desde o Senegal até Angola; Pacífico Oriental: desde a Baixa Califórnia até o Peru; Pacífico Central: Ilhas Clipperton e Galápagos.

\section{Cronius tumidulus (Stimpson, 1971)}

RATHBUN, 1930: 142.

Material. Amapá, Maranhão, Ceará, Rio Grande do Norte, Paraíba, Pernambuco, Alagoas; recifes; fundos de lama ou de algas calcárias; 0-85m, infralitoral e circalitoral. Bahia.

Distribuição geográfica. Atlântico Ocidental: desde as Antilhas até a

\section{Portunius Weber, 1795}

Chave para identificação das espécies

1. Carapaça larga, isto é, margens ântero-laterais constituindo o arco de um círculo cujo centro está localizado perto da margem posterior da

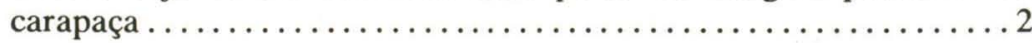

Carapaça estreita, isto é, margens ântero-laterais constituindo um círculo cujo 
centro está localizado na região cardíaca $\ldots \ldots \ldots \ldots \ldots \ldots \ldots \ldots$

2. Superfície inferior da carapaça com uma carena estridulante ...... vocans

- Superfície inferior da carapaça desprovida de carena estridulante ...... 3

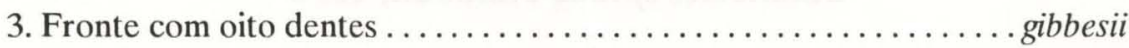

- Fronte com seis dentes . . . . . . . . . . . . . . . . . . . . . 4

4. Margem póstero-distal do mero das patas natatórias com um espinho forte . rufiremus

- Margem póstero-distal do mero da patas natatórias desprovido de espinhos ou espínulos .................................. 5

5. Margem externa do mero dos quelípedes com um espinho junto da extremidade anceps

- Margem externa do mero dos quelípedes desprovida de espinho junto da extremidade ventralis

6. Margem póstero-distal do mero das patas natatórias sem espínulos ou espinhos floridanus

- Margem póstero-distal do mero das patas natatórias com espínulos ou es-

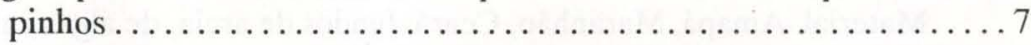

7. Margem póstero-distal do mero das patas natatórias com um espínho, além

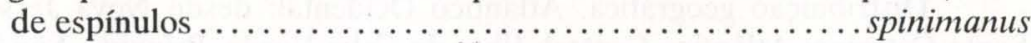

- Margem póstero-distal do mero das patas natatórias apenas com espínulos .. .8

8. Espinho interno do carpo dos quelípedes mais longo que a palma

spinicarpus

Espinho interno do carpo dos quelípedes nitidamente mais curto que a palma ordwayi

\section{Portunus anceps (Saussure, 1858)}

WILLIAMS, 1984: 387.

Material. Amapá, Pará, Maranhão, Ceará, Pernambuco, Alagoas; fundos de lama, de areia ou de algas calcárias; 0-130m, infralitoral, circalitoral e batial.

Distribuição geográfica. Atlântico Ocidental: desde a Carolina do Norte até a Bahia; Atlântico Central: Bermudas.

\section{Portunus floridanus Rathbun, 1930 *}

WILLIAMS, 1984: 387.

Distribuição geográfica. Atlântico Ocidental: desde a Carolina do Norte 
até as Guianas.

\section{Portunus gibbesii (Stimpson, 1859 *}

WILLIAMS, 1984: 389.

Material. Maranhão.

Distribuição geográfica. Atlântico Ocidental: desde Massachussetts até o Maranhão.

$$
\text { Portunus ordwayi (Stimpson, 1860) }
$$

WILLIAMS, 1984: 390.

Material. Pará, Maranhão, Piauí, Ceará, Rio Grande do Norte, Pernambuco, Alagoas, Fernando de Noronha; fundos de areia ou de algas calcárias; $0-90 \mathrm{~m}$, infralitoral e circalitoral.

Distribuição geográfica. Atlântico Ocidental: desde Massachussetts até o Rio de Janeiro; Atlântico Central: Bermudas, Fernando de Noronha.

\section{Portunus spinicarpus (Stimpson, 1871)}

WILLIAMS, 1984: 392.

Material. Amapá, Pará, Alagoas, Bahia, Espírito Santo, Rio de Janeiro, São Paulo; fundos de lama, de areia e detríticos; 36-116m, circalitoral e batial.

Distribuição geográfica. Atlântico Ocidental: desde a Carolina do Norte até Santa Catarina.

\section{Portunus spinimanus Latreille, 1819}

WILLIAMS, 1984: 393.

Material. Maranhão, Pernambuco, Alagoas, Rio de Janeiro, Rio Grande do Sul.

Distribuição geográfica. Atlântico Ocidental: desde a Carolina do Norte até o Rio Grande do Sul. Atlântico Central: Bermudas.

\section{Portunus rufiremus Holthuis, 1959}

HOLTHUIS, 1959: 195.

Material. Amapá, Pará; fundos de lama ou de areia; $21-70 \mathrm{~m}$, circalitoral. Pará.

Distribuição geográfica. Atlântico Ocidental: desde as Guianas até o

Portunus ventralis (A. Milne Edwards, 1879)

RATHBUN, 1930: 43.

Material. Rio Grande do Norte, Alagoas; fundos de algas calcárias; 25-40m, infralitoral. 
Distribuição geográfica. Atlântico Ocidental: desde a Geórgia até as Antilhas e desde o Rio Grande do Norte até o Rio de Janeiro.

\section{Portunus vocans (A. Milne Edwards, 1878)}

RATHBUN, 1930: 60 .

Material. Bancos ao largo do Espírito Santo; fundos de algas calcárias; 61-70m, circalitoral.

Distribuição geográfica. Atlântico Ocidental: Antilhas; Atlântico Central: bancos ao largo do Espírito Santo e Ilhas de Ascenção, Cabo Verde, Príncipe, São Tomé e Ano Bom.

\section{REFERÊNCIAS BIBLIOGRÁFICAS}

BOSCHI, E.E. 1964. Los crustaceos decapodos Brachyura del litoral Bonaerense. Bol. Inst. Biol. Marina 6: 1-76.

COELHO, P.A. \& M.A. RAMOS. 1972. A constituição e a distribuição da fauna de decápodos do litoral leste da América do Sul entre as latitudes de $5^{\circ} \mathrm{N}$ e $39^{\circ} \mathrm{S}$. Trab. Oceanogr. Univ. Fed. Pernambuco 13: 133-236.

HOLTHUIS, L.B. 1959. The Crustacea Decapoda of Suriname (Dutch Guiana). Zool. Verhand. 44: 1-126.

- 1991. Marcgraf's (1648) Brazilian Crustacea. Zool. Verhand. 268: 1-123.

RATHBUN, M.J. 1930. The cancroid crabs of America of the families Euryalidae, Portunidae, Atelecyclidae, Cancridae and Xanthidae. Bull. U. S. Nat. Mus. 152: 1-609.

SEBA, A. 1759. Locupletissimi rerum naturalium thesauri accurata descriptio et iconibus artificiosissimis expressis per universam physices historiam. 3 , 212p.

STEPHENSON, W. \& M. REES. 1968. A revision of the genus Ovalipes Rathbun, 1898 (Crustacea, Decapoda, Portunidae). Rec. Austr. Mus. 27 (11): 213-261.

WILLIAMS, A.B. 1984. Shrimps, lobsters and crabs of Atlantic coast of

Eastern United States, Maine to Florida. Washington, Smithsonian Institution Press, 550p.

Recebido em 23.IX.1992; aceito em 19.VIII.1993. 